\title{
Laparoscopic surgical transmesocolic jejunostomy: A new surgical approach
}

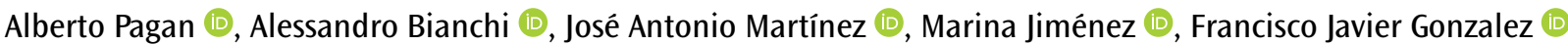

In cancer patients with tumors of the upper gastrointestinal tract, dysphagia and cachexia require gastrostomy or jejunostomy as the only options for enteral access for long-term feeding. In this article, the authors describe a modified placement of laparoscopic feeding jejunostomy applied during laparoscopic oncology layering technique. After performing an exploratory laparoscopy, a feeding jejunostomy is performed using a Foley silicon catheter, through an eyelet in the mesentery of the descending colon. After completing the introduction of the jejunal probe according to the Witzel technique, the intestinal segment of jejunum is attached to the internal sheath of the mesocolon using sutures polysorb $2 / 0$, with the aim of removing the possible internal hernia and a jejunal torque that could cause an intestinal obstruction. There were no intraoperative complications or mortality. The technique described here provides most of the benefits of laparoscopic jejunostomy feeding, avoiding the possible internal hernia.

Keywords: Jejunostomy, enteral nutrition, upper gastrointestinal tumors
\end{abstract}

ORCID IDs of the authors: A.P. $0000-0001-8142-0952$; A.B. 0000-0002-4571-0511; J.A.M.0000-0002-7193-9360; M.J. 0000-0002-3085-5793; F.J.G. 0000-0003-0003-9055.

\section{Cite this paper as:} Pagan A, Bianchi A, Martínez $J A$, Jiménez $M$, Gonzalez XF. Laparoscopic surgical transmesocolic jejunostomy: a new surgical approach. Turk J Surg 2018; 34: 155-157.

Department of General Surgery, Hospital Universitario Son Espases, Palma De Mallorca, Spain

\section{Corresponding Author} Alessandro Bianchi e-mail:aplbianchi@gmail.com

Received: 26.05.2015 Accepted: 29.08.2015 Available Online Date: 03.01.2018

CCopyright 2018 by Turkish Surgical Association Available online at www.turkjsurg.com

\section{INTRODUCTION}

Nutrition in patients with cancer of the upper gastrointestinal tract is essential for a good quality of life. Often patients suffering from this type of cancer are malnourished or cachectic at the time of diagnosis. In these patients, feeding plays a crucial role, even as part of a palliative treatment. Several studies have shown the benefits of enteral feeding over parenteral feeding. Depending on the location of the tumor and the clinical stage, there are several options available for the administration of enteral feeding. Since the introduction of percutaneous endoscopic gastrostomy and gastrostomy placed under radiographic control, surgical techniques such as gastrostomy or jejunostomy have fewer indications. However, when the new techniques described above cannot be performed, surgery is again useful and essential. Most surgical techniques are used in patients with tumors of the upper gastrointestinal tract, head, and neck where the noninvasive techniques are impossible or dangerous to perform. In these patients, open gastrostomy and jejunostomy are the only option for enteral access. Since the first report of laparoscopic jejunostomy described by O'Regan in 1990, there have been several technical publications of laparoscopic feeding jejunostomy. The existence of multiple procedures indicates that there is no standard procedure (1).

\section{CASE PRESENTATIONS}

\section{Clinical Cases}

Case 1: A 58-year-old male patient with an adenocarcinoma of the gastric antrum presented food intolerance and weight loss of nearly $15 \mathrm{~kg}$ in 2 months. In the extension study, the gastroscopy reported the presence of abundant food remains and proliferative and ulcerated lesion in antral region that did not allow the passage of an endoscopic tube. The biopsy of the ulcerated lesion was positive for intestinal type adenocarcinoma. Computed tomography scan showed lymphadenopathy in the left cardial region and lesser gastric curvature of $12 \mathrm{~mm}$ and objectified gastric wall thickening with signs of externalization. Exploratory laparoscopy was indicated. Peritoneal lavage was negative for tumor cells. Because of this, a feeding jejunostomy was placed.

Case 2: A 62-year-old female patient was diagnosed with gastric linitis and weight loss of $17 \mathrm{~kg}$ in 5 months. Gastroscopy showed submucosal infiltration from the cardia to the antrum. Endoscopic biopsies were negative. The echo-endoscopy showed a submucosal thickening of approximately $1 \mathrm{~cm}$. Due to severe dysphagia and cachexia, laparoscopic jejunostomy placement for enteral feeding was performed.

Preoperative period: Informed consent was obtained in accordance with local regulations for both patients. Standard perioperative antibiotic prophylaxis was carried out with $2 \mathrm{~g}$ of amoxicillin clavulanic Ev 30 min before surgery. Antithrombotic prophylaxis was applied including mechanical and pharmacological prophylaxis with low molecular weight heparin LMWH (40 mg of enoxaparin sodium). 
Position of patient: The procedure was performed under general anesthesia. The patient was placed in prone position with arms outstretched to the sides. The operator was placed between the patient's legs, the first assistant on the patient's right side and the scrub nurse on the left side of the patient.

Trocar placement: Two 11-mm trocars and a 5-mm trocar were used. Pneumoperitoneum was performed through a Verres needle inserted in left hypochondrium. A pneumoperitoneum of $12 \mathrm{mmHg}$ of $\mathrm{CO} 2$ was achieved. The first trocar was placed in the middle left clavicular line. The remaining trocars were placed depending on the anatomical characteristics of the patient.

\section{Surgical Technique}

Exploratory laparoscopy was performed using an optical $30^{\circ} \mathrm{cam}-$ era. For the inspection of the abdominal cavity, for assessing the size of the tumor and its spread, and the possible presence of metastases were used standard tools were used.Peritoneal lavage is performed to rule out the peritoneal spread of tumor cells. Samples are taken for pathologic examination and to detect HER2 receptors.

A loop of jejunum was selected at a distance of $20 \mathrm{~cm}$ from the Treitz ligament. The descending colon was set to be free from the left parietocolic wall and a little hole was made in the left mesenteric leaf. A small incision was made in the abdominal wall, in correspondence to the anterior axillary line.In the planned point, jejunum was opened and a Foley catheter was introduced and placed in accordance with Witzel technique. The permeability of the catheter balloon was checked and filled with $2 \mathrm{ml}$ of distilled water. Jejunal segment was fixed to the mesenteric sheet of the descending colon.

\section{Results}

There were no intraoperative complications. Mean operative time was approximately $45 \mathrm{~min}$. There was no blood loss. The short-term outcome was good. There were no postoperative complications or mortality. The use of the jejunostomy was initiated within the first 24 postoperative hours. Then patients were transferred to the oncology unit where underwent the neoadjuvant treatment. The results of the peritoneal fluid tests showed no cancer cells in both patients. Based on the intraoperative findings, the first patient received a neoadjuvant treatment and then a laparoscopic subtotal gastrectomy was performed. The second patient was treated by palliative treatment.

\section{DISCUSSION}

Malnutrition and cachexia are often symptoms of tumors of the upper gastrointestinal tract. In unresectable or resectable carcinomas of the head and neck, surgery and radiochemotherapy are routinely performed; therefore, the use of enteral prophylactic approach, as jejunostomy, allows improving the quality of life of patients and prevents the complications of malnutrition during treatment. The progress of noninvasive techniques has shifted the surgical approach. In rare cases, when there is an obstruction of the oropharyngeal and esophageal region, the use of surgical techniques is required to place a jejunostomy catheter or to perform gastrostomy. The procedures performed at present are Witzel jejunostomy, needle catheter jejunostomy, jejunostomy through a serous tunnel, and jejunostomy in Roux-Y. The multiplicity of procedures, essentially reflect the need to find an optimal method as the literature describes (1).
Despite the advantages of feeding by a jejunostomy, serious complications, such as internal hernia and intestinal volvulus, have been described-complications that can be life threatening. Because of this, jejunostomy placement has been questioned by some authors (2); however, other authors (3), knowing its complications, systematically placed the jejunostomy tube during upper gastrointestinal surgery for postoperative enteral nutrition. The frequency of major surgical complications varies from $2.1 \%$ to $3.6 \%$. One of this is the intestinal obstruction due to a volvulus around the jejunostomy tube site. In a large study, intestinal obstruction and volvulus occurred in $0.14 \%$ patients (4).

The aim of this study was to describe a surgical technique for the jejunostomy placement during laparoscopic staging of two consecutive patients with advanced cancer of the upper gastrointestinal tract. The technique presents the same benefits of the laparoscopic approach, as an early reintroduction of the enteral diet. Furthermore, this technique does not limit a second surgery, such as a total gastrectomy, especially in the use of the afferent loop, and reduces a possible serious complication related to the formation of an internal hernia.

The main drawback of this method is due to the lateralization of the jejunostomy tube that could limit patient autonomy. According to us, the tunneling of the jejunostomy catheter to the anterior abdominal wall would minimize this effect.

\section{CONCLUSION}

This new laparoscopic transmesocolic approach is as feasible as other minimally invasive techniques and can be used during a staging surgery and when a need for nutritional support is required. Transmesocolic approach permits the prevention of internal hernia and intestinal volvulus, the two main complications related to the placement of a jejunostomy. Our proposal is to perform an anchorage of the jejunum to peritoneal sheet of the descending mesocolon that involves the parietal fixing of the probe of the jejunostomy. Further studies in larger groups of patients are needed to evaluate the long-term results of this procedure.

Informed Consent: Written informed consent was obtained from patients who participated in this study.

Peer-review: Externally peer-reviewed.

Author Contributions: Concept - A.P.; Design - A.P., A.B.; Supervision - X.F.G.; Resource - A.P., A.B.; Materials - A.B.; Data Collection and/or Processing - A.B., M.J.; Analysis and/or Interpretation - A.P., A.B.; Literature Search - A.B., J.A.M., M.J.; Writing Manuscript - A.P., A.B.; Critical Reviews - X.F.G.

Acknowledgements: The technical assistance of the nurse staff of the General Surgery Department is gratefully acknowledged.

Conflict of Interest: The authors have no conflicts of interest to declare.

Financial Disclosure: The authors declared that this study has received no financial support. 


\section{REFERENCES}

1. Kirby DF, Flemming CR. American Gastroenterological Association Technical Review on Tube Feeding for Enteral Nutrition. Gastroenterology 1995; 108: 1282-1301. [CrossRef]

2. Zapas JL, Karakozis S, Kirkpatrick JR. Prophylactic jejunostomy: a reappraisal. Surgery 1998; 124: 715-719. [CrossRef]
3. Srinathan SK, Hamin T, Walter S, Tan AL, Unruh HW, Guyatt G. Jejunostomy tube feeding in patients undergoing esophagectomy. Can J Surg 2013; 56: 409-414. [CrossRef]

4. Myers JG, Page CP, Stewart RM, Schwesinger WH, Sirinek KR, Aust JB. Complications of needle catheter jejunostomy in 2,022 consecutive applications. Am J Surg 1995; 170: 547-551. [CrossRef] 